\title{
Development of Switching Performance Evaluator and Arc Modelling Tool for Low-Voltage Switching Devices
}

\section{Abstract}

\section{Purpose}

The main objective of this study is to investigate a reliable evaluator of arc re-ignition and to develop a numerical tool for accurate prediction of arc behaviour of low-voltage switching devices (LVSDs) prior to empirical laboratory testing of real products.

\section{Design/methodology/approach}

Two types of interruption tests have been carried out in the investigation of re-ignition evaluators. Arc modelling tool coupled with the load circuit has been developed to predict arc characteristics based on conventional magnetohydrodynamics theory, with special attention given to Lorentz force acting on the arc column and surface phenomena on the splitter plate. The model assumptions have been validated by experimental observation of arc motion and current and voltage waveforms.

\section{Findings}

It is found that the exit-voltage across the switching device and the ratio of system to exit-voltage at the current zero point are reliable evaluators for prediction of re-ignition. Where the voltage ratio is positive, instantaneous re-ignition does not occur. Further, the probability of re-ignition is very low if the voltage ratio is in the rage of 1.3 to 0 .

\section{Originality/value}

It is observed that the voltage ratio can be considered as a reliable global evaluator of re-ignition, which can be used for various types of LVSD test conditions. In addition, it is shown that arc modelling allows a good prediction of the current and voltage waveforms, arc motion as well as the exit-voltage, which can be used to obtain the evaluator of re-ignition.

\section{Introduction}

Low-voltage switching devices (LVSDs) are essential to turn on and off electric current and to protect humans and other connected equipment against overload or short circuit accidents in the power distribution network. A quenching chamber of an LVSD is the main volume for switching current and it consists of a movable and fixed contact, magnetic yoke, arc runner, vent and splitter plates (see Figure 1). When the movable contact separates from the fixed contact, an arc is established between the contacts and it moves towards the splitter plates by gas flow and Lorentz force. Concurrently, there is a dramatic increase in the arc voltage due to the multiple anodic and cathodic potential drops in surfaces of the plates. Ideally, the arc is extinguished at the first current zero moment, however the arc can re-ignite beyond this point. During this breaking process, the arc parameters have a great influence on interruption performance of the LVSD (Freton and Gonzalez, 2009).

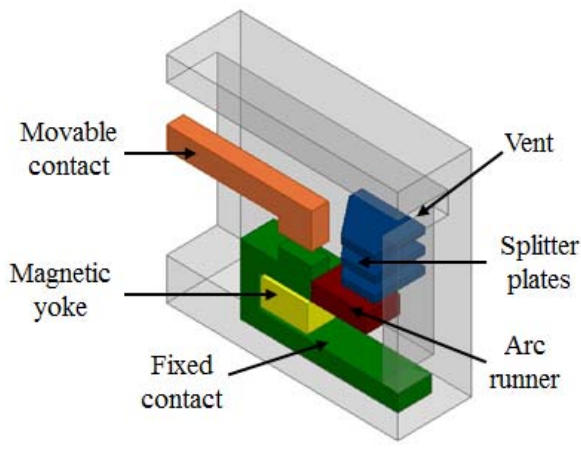

Figure 1. Half symmetric schematic structure of a quenching chamber in an LVSD.

There has been several reports on correlations between the experimentally observed behaviour of the arc and predicted performance of LVSDs. McBride et al. carried out experimental studies of the influence of contact opening velocity and material, wall material and venting condition on the arc motion in a miniature circuit breaker using a fibre optic imaging system, pressure gauges and spectrograph (McBride et al., 2002). Balestrero 
et al. introduced several 'microscopic evaluators' that can predict re-ignition by measuring the current or arc voltage over a $10 \mu \mathrm{s}$ time period near the current zero event, when ion recombination and non-equilibrium phenomena dominate (Balestrero et al., 2010). Hauer et al. found that the probability of re-ignition after the current zero event is heavily dependent on the 'exit-voltage' (the arc voltage immediately prior to the current zero event) and Shin et al. backed Hauer's findings by showing the test results of different types of LVSDs (Hauer and Zhou, 2014, Shin et al., 2015, 2016).

Arcs are nonlinear phenomena and their characteristics are strongly dependent on the dimensions, temperature, pressure and attachment points of the arc. For the reliable prediction of the switching performance, the arc behaviour should be accurately simulated. Karetta et al. analysed the arc motion with a 3-D magnetohydrodynamics (MHD) model incorporating heat conduction, gas and current flows and magnetic force (Karetta and Lindmayer, 1998). Lindmyer et al. extended MHD arc modelling for the arc root formation by introducing the nonlinear relationship between the potential drop and current density in the arc root region of the splitter plates (Lindmayer et al., 2006). Rong and Ma et al. conducted numerical analysis on the influence of metal erosion and wall ablation on plasma properties and arc behaviour in an LVSD (Rong et al., 2009, Ma et al., 2009).

Although the technology available for the design and analysis of LVSDs has been notably developed thanks to the previous experimental and numerical studies, there are still limitations in the prediction of the switching performance and optimization of LVSDs. The experimental approach is expensive and time-consuming. Moreover, it is difficult to experimentally obtain internal arc parameters such as current density and temperature that are useful in improving the design. Most previous numerical methods have focused on the behaviour of the arc plasma prior to the current zero moment without evaluating the probability of re-ignition following the current zero moment, even though avoiding re-ignition is a key goal when designing the quenching chamber.

This paper presents a reliable evaluator of switching performance, which can helps judge the probability of reignition and a numerical tool for accurate prediction of arc behaviour in the LVSDs.

\section{Experiments on arc re-ignition}

It is considered that an interruption trial is successful without re-ignition if the breakdown voltage in the switch is greater than recovery voltage (Slade, 2014, Slepian, 1928); however, the evaluator is required for a reignition prediction for designing LVSDs because of the complexity in accurately calculating the breakdown voltage of the arc plasma in an LVSD after the current zero moment. It changes with time and depends on geometry due to complex recombination processes taking place inside the switch. In this paper, the re-ignition evaluator has been obtained prior to the current zero point without complex calculation of the breakdown voltage and it is used as a parameter which can judge the arc phenomena (re-ignition) after the current zero point.

\subsection{Experimental method and explanation of switching circuit}

The experimental investigation was carried out through two types of interruption tests (see Table I). The first is a low power test for a single-pole miniature circuit breaker (MCB) and the second is a high power one for a three-pole moulded case circuit breaker (MCCB). Figure 2 shows test circuits for the interruption tests of singlepole and three-pole LVSDs. The current and voltage waveforms were recorded by an oscilloscope during switching tests. All tests were conducted with a previously unused switching device and every device has a different quenching chamber design (vent size and position, splitter plate's geometry, etc.) or contact opening velocity.

Table I. Two types of interruption tests.

\begin{tabular}{|c|c|c|c|c|c|}
\hline $\begin{array}{c}\text { Test } \\
\text { sample }\end{array}$ & Number of pole & Voltage [V] & $\begin{array}{c}\text { Prospective current } \\
{[\mathrm{A}]}\end{array}$ & Power factor & $\begin{array}{c}\text { Frequency } \\
{[\mathrm{Hz}]}\end{array}$ \\
\hline MCB & 1 & 252 (phase voltage) & 10000 & 0.45 & 60 \\
\hline $\mathrm{MCCB}$ & 3 & $\begin{array}{c}483 \\
\text { (line-to-line voltage) }\end{array}$ & 20000 & 0.3 & 60 \\
\hline
\end{tabular}




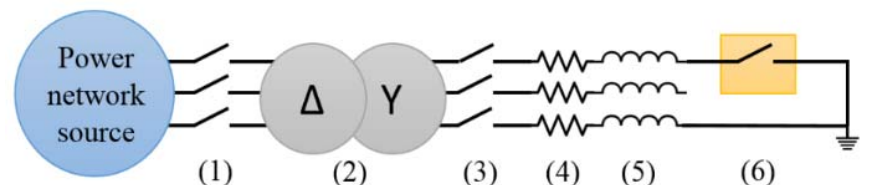

(1)

(2)

(3)

(4) (5)

(6)

(a) Single-phase test for MCBs

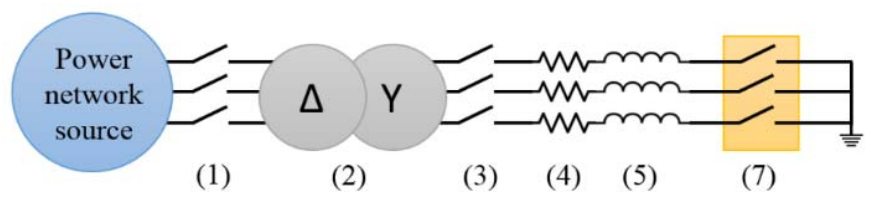

(b) Three-phase test for MCCBs

Figure 2. Test circuits for a single-phase and three-phase test: (1) back-up circuit breaker, (2) three-phase transformer, (3) making switch, (4) resistor, (5) reactor, (6) test MCB, (7) test MCCB.

Figure 3 shows the equivalent circuit during the interruption process. During switching operation of the LVSD, the arc current is significantly reduced due to the arc voltage when compared to the prospective current that is determined by the resistance $(\mathrm{R})$, reactor $(L)$ and system voltage $\left(U_{s}\right)$. This current limitation is explained by equation (1). The arc voltage is gradually raised up as the arc moves towards the splitter plates after the ignition between contacts. The arc voltage plays a role as a resistor to prevent the arc from increasing rapidly. Further, when the arc voltage is higher than the system voltage the rate of change of the current $(d I / d t)$ becomes negative and it leads to the early current zero point.

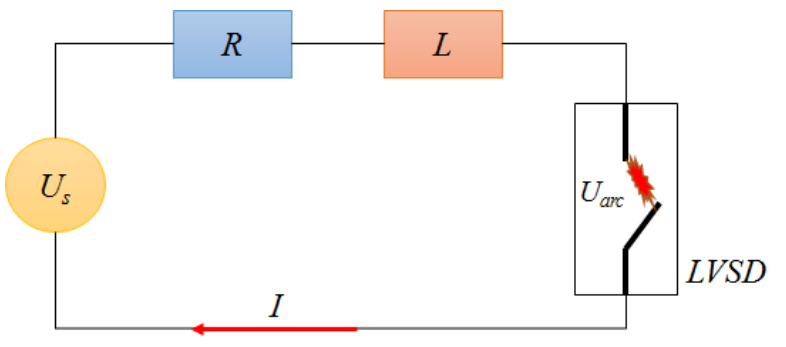

Figure 3. Equivalent circuit during interruption process; $U_{s}$ is the system voltage, $I$ is the current, $R$ is the resistance, $L$ is the inductance and $U_{\text {arc }}$ is the arc voltage.

$$
U_{S}(t)=I(t) R+L \frac{d I(t)}{d t}+U_{a r c}(t)
$$

\subsection{Experimental results and discussion}

Figure 4 shows the voltage and current waveforms for both a successful and a failed interruption of an MCB. A successful interruption means that the short circuit current is interrupted at the first current zero point, whereas a failed one indicates there is re-ignition after the current zero point. During a successful interruption, the arc voltage waveform is relatively high and remains so up until the current zero point when compared to the voltage waveform of a failed interruption. If the LVSD fails to interrupt the short circuit current at the first current zero moment, a large current continues to flow until at least the next current zero point resulting in severe damage to the device. 


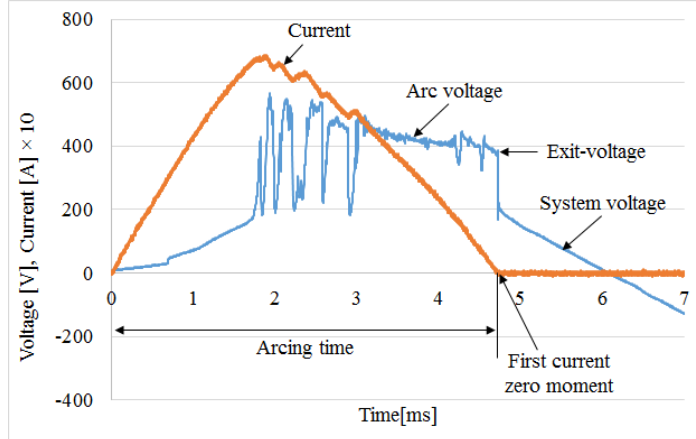

(a) Successful interruption test

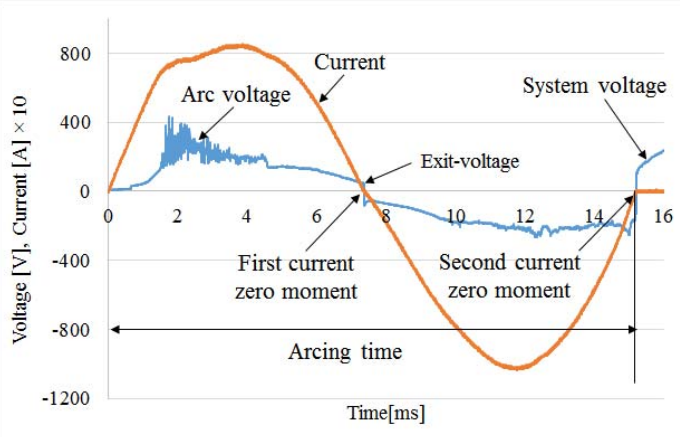

(b) Failed interruption test

Figure 4. Voltage and current waveforms during interruption operation of MCBs.

Table II shows some switching test results of MCBs. System voltage means the value of system voltage at the current zero moment and re-ignition voltage is the value of arc voltage immediately after the current zero point when re-ignition happens. There are two possible cases of polarity: the exit-voltage and system voltage may have the same polarities (test number 3) or opposing polarities (test number 1, 8 and 22). In addition, re-ignition can be further classified as one of two types [4]: 'instantaneous' re-ignition, which occurs immediately after the current zero event causing the short circuit current continues to flow in reverse polarity and 'delayed' re-ignition, where there is a pause between the current zero moment and re-ignition (Hauer and Zhou, 2014).

Table II. Some test results of MCBs.

\begin{tabular}{|c|c|c|c|c|c|}
\hline $\begin{array}{c}\text { Test } \\
\text { number }\end{array}$ & $\begin{array}{c}\text { Exit-voltage } \\
{[\mathrm{V}]}\end{array}$ & $\begin{array}{c}\text { System voltage } \\
{[\mathrm{V}]}\end{array}$ & $\begin{array}{c}\text { Re-ignition } \\
\text { Voltage [V] } \\
\text { immediately after the } \\
\text { current zero point })\end{array}$ & Polarities & Remark \\
\hline 1 & 87 & -103 & - & Different & Success \\
\hline 3 & 315 & 123 & - & Same & Success \\
\hline 8 & 53 & -198 & -125 & Different & $\begin{array}{c}\text { Instantaneous } \\
\text { fail }\end{array}$ \\
\hline $22 *$ & 87 & $\begin{array}{c}-73 \\
(-265 \text { at delayed } \\
\text { re-ignition point })\end{array}$ & -115 & Different & Delayed fail \\
\hline
\end{tabular}

*The delayed re-ignition occurs at the moment of $1.49 \mathrm{~ms}$ after the first current zero point. Note that the reignition voltage (the arc voltage immediately after the current zero point) is different from the exit-voltage due to plasma cooling and expansion.

\subsubsection{Exit-voltage as a re-ignition evaluator}

Figure 5 presents the relationship between occurrence of arc re-ignition and the exit-voltage at the first current zero moment in the MCB and MCCB tests. It can be seen there are different thresholds of the exit-voltage that distinguish successful interruptions from failed interruptions, which are around $80 \mathrm{~V}$ for MCB tests and about $133 \mathrm{~V}$ for MCCB tests. These test results illustrate that re-ignition is strongly correlated to the exit-voltage but the threshold value varies with the test condition. 


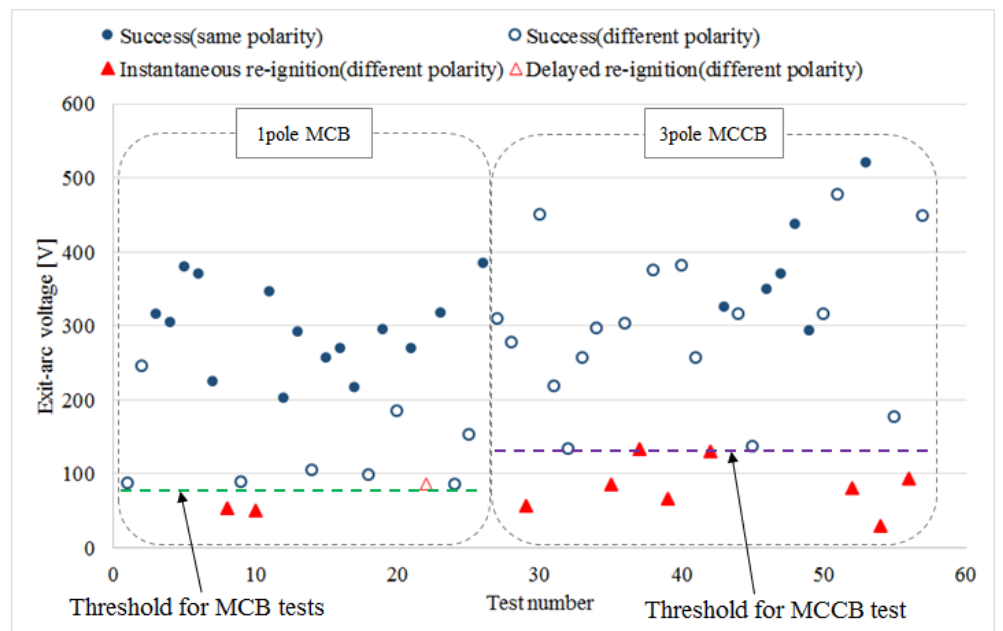

Figure 5. Relationship between interruption performance and exit-arc voltage of MCBs and MCCBs.

\subsubsection{Voltage ratio as a re-ignition evaluator}

In order to investigate a reliable evaluator for predicting re-ignition, the concept of the voltage ratio is introduced. The voltage ratio is calculated from the exit-voltage and the system voltage near the current zero, given in equation (2)

$$
\text { Voltage ratio }=U_{s}\left(t_{0}\right) / U_{\text {exit }},
$$

where $t_{0}$ is the instant of the first current zero, $U_{s}$ is the system voltage and $U_{\text {exit }}$ is the exit-voltage.

Figure 6 shows the dependence of interruption performance of MCBs and MCCBs on the voltage ratio at the first current zero moment. It can be seen that the switching trials are always successful in the case of the same polarity (positive voltage ratio). Of the 26 trials where the voltage ratio is in the range of -1.3 to 0 , there is only a single delayed re-ignition, not predicted by the exit-voltage or voltage ratio evaluators. This anomaly may be attributed to a fault in the operating mechanism detected during the post-test examination of the device.

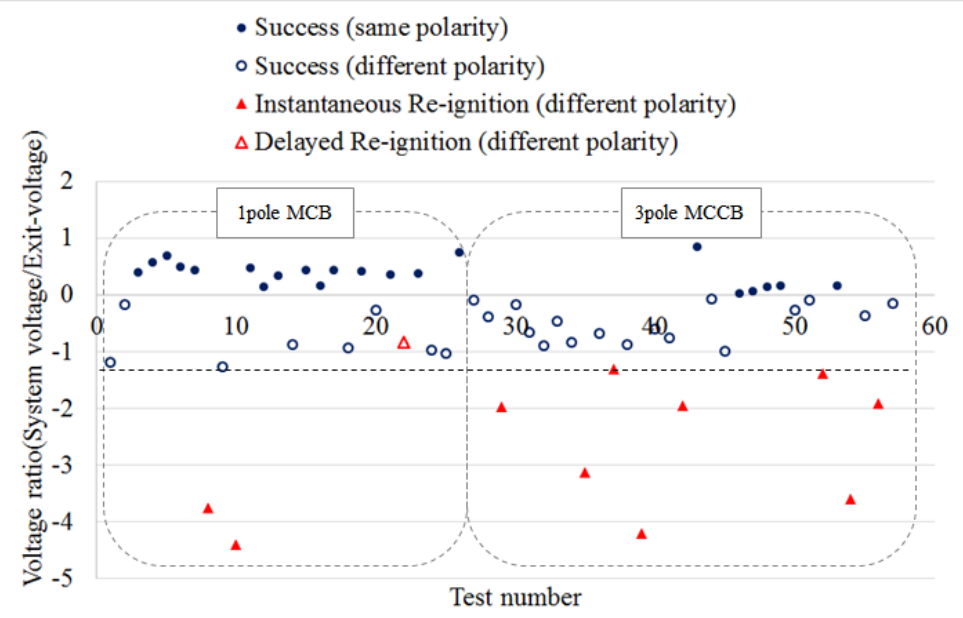

Figure 6. Relationship between the interruption performance and the voltage ratio of MCBs and MCCBs.

It is interesting to note that the interruption test is always successful if the voltage ratio is positive (the exitvoltage has the same polarity as the system voltage). This situation happens only when the arc moves quickly towards the splitter plates and a high arc voltage is generated. This result can be explained by Figure 3 and equation (1). Solving (1) at an instant $t$ near the current zero point, the voltage drop across the external resistive load, the first term of the right hand side, is negligible and the circuit equation can be written as

$$
U_{S}(t)-U_{a r c}(t)=L \frac{d I(t)}{d t} .
$$


If instantaneous re-ignition can occur in the same polarity case (with decreasing current) where both system voltage and exit-voltage are initially positive, the value of the left hand side in (3) prior to the current zero moment is negative, but switches to positive immediately after the current zero moment due to the inversion of the arc voltage to negative. However, the value of the right hand side is still negative because the current continues to decrease after the current zero moment. It indicates that such situations are not possible and the only solution is an open circuit, i.e. successful interruption.

On the other hand, when the system voltage is negative but the exit-voltage is positive (in the case of different polarity), it may support the negative e.m.f. ( $L \frac{d I(t)}{d t}$ ) and provide a sufficient power for re-ignition to occur. After the current zero point, both the system and arc voltages are negative. In order to support any current flow and negative e.m.f., the absolute value of the system voltage should be higher than that of the re-ignition voltage (arc voltage immediately after the current zero point). Figure 7 fits with this explanation by showing the ratio of the system voltage to the re-ignition voltage when instantaneous re-ignition happens. Furthermore, a linear trend is observed between the absolute values of the re-ignition voltage and exit-voltage (Figure 8), which means that the exit-voltage considerably affects the arc characteristics (re-ignition voltage) after the current zero moment. This is the reason why the voltage ratio can also be a re-ignition evaluator in the case of different polarity and the reignition probability is very rare if the voltage ratio lies between around -1.3 and 0 .

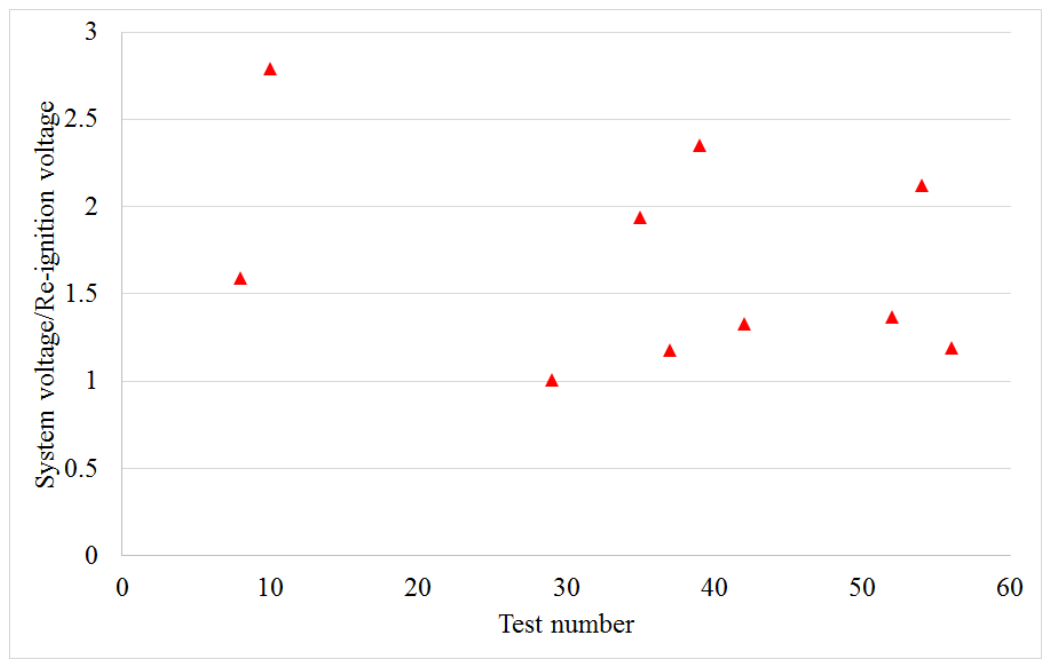

Figure 7. Ratio of the system voltage to re-ignition voltage for non-successful interruption tests.

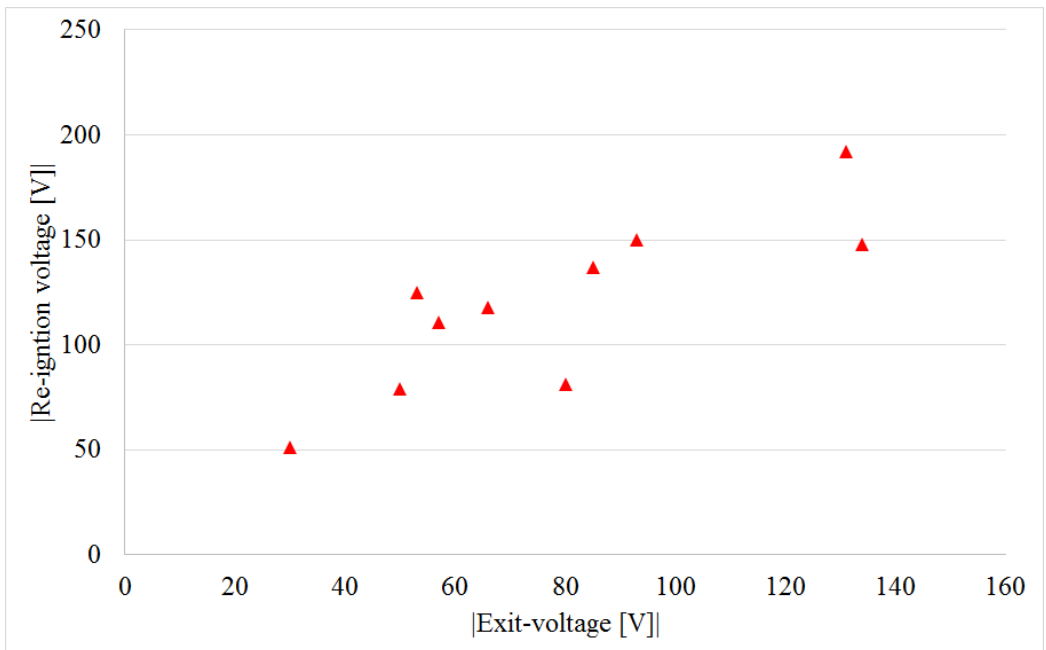

Figure 8. Relationship between the absolute values of the re-ignition voltage and exit-voltage for non-successful interruption tests: re-ignition voltage is higher than exit-voltage due to increased plasma resistance (cooling and expansion effects), and the error median between the absolute values of the re-ignition voltage and exit-voltage is $61.3 \%$. 


\section{Numerical model and simulation results}

Prior to MHD computation of the arc plasma, Lorentz force is calculated by finite element method (FEM) and used as an input parameter (the external magnetic flux density). The effect of the arc root region is modelled using the contact resistance on the surface of the plate. To validate the model, the simulated motion is compared with experimentally measured arc motion by using a high speed optical arc imaging system (AIS) connected to a switching test apparatus described below.

\subsection{Arc image measurement for validation of arc model}

The flexible test apparatus (FTA) in Figure 9 is designed as the structure of an MCB and it is used to record the arc motion under controlled test conditions (McBride et al., 2002, 2016). There are 109 fibres distributed in the quenching chamber including 32 fibres between the splitter plates, which have $1 \mathrm{MHz}$ sampling rate. The optic fibres are slotted into the holes in the fibre array block. The photosensors of the AIS have a spectral response range from $320 \mathrm{~nm}$ to $1060 \mathrm{~nm}$ and it enables the tracking of the arc motion through the light intensity and position in the chamber during an interruption operation. A half cycle wave of a short circuit current is provided by a capacitor bank and its magnitude is changed by adjusting a charging voltage of a capacitor.

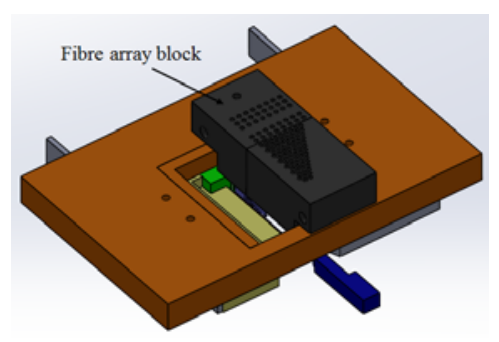

(a) External configuration

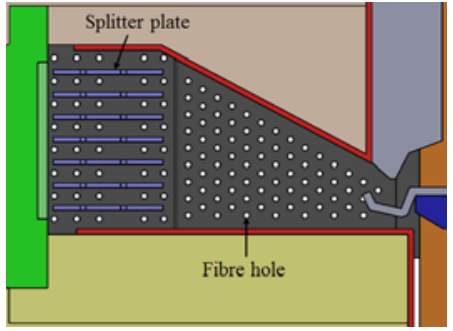

(b) Internal configuration

Figure 9. The flexible test apparatus for the arc image measurement.

\subsection{Calculation of Lorentz force}

Lorentz force is calculated by a commercial FEM software depending on the current (from 0 A to 2000 A) and arc position (from the ignition region to splitter plates). Figure 10 shows the analysis geometry of Lorentz force. Figure 11 presents some results of Lorentz force acting towards the splitter plates depending on the current and arc column position (these data are used to obtain the external magnetic flux density that varies with the current and arc position).

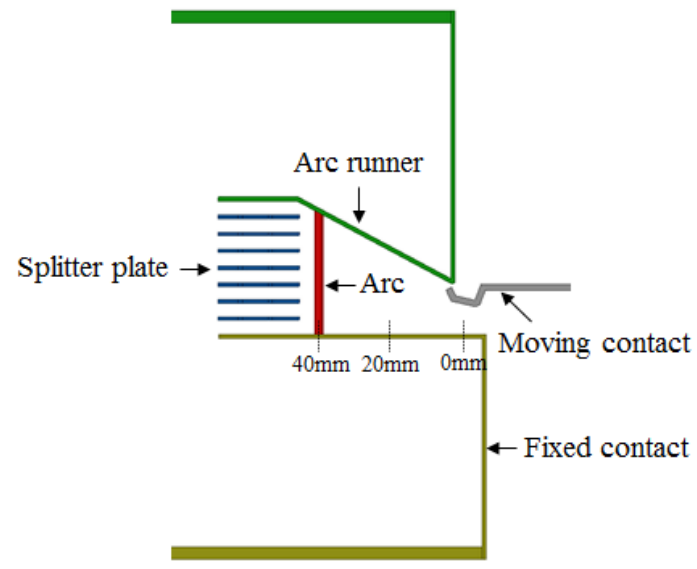

Figure 10. The Analysis model of Lorentz force. 


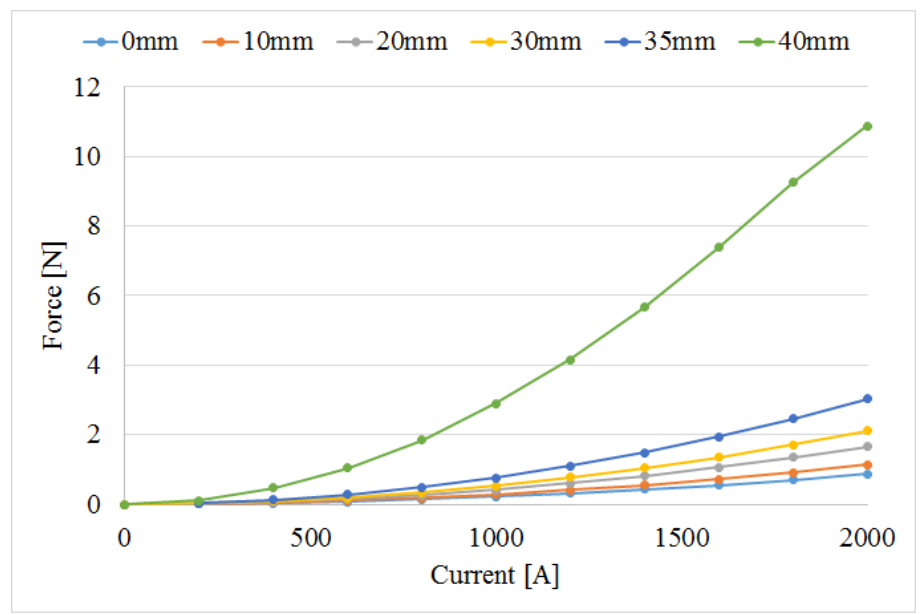

Figure 11. Lorentz force acting towards the splitter plates depending on the current and arc position.

To consider the influence of Lorentz force on the arc motion, the external magnetic flux density $B_{e x}$ is computed by (4), and (5)

$$
\begin{gathered}
F_{L o}=I B_{e x} L, \\
B_{e x}=F_{L o} /(I L),
\end{gathered}
$$

where $F_{L o}$ is Lorentz force (data in Figure 11), $I$ is the current, and $L$ is the arc length at the specific position (the arc column is assumed to be perpendicular to the fixed contact in the quenching chamber). In the arc model (MHD computation), $B_{e x}$ is used as the input parameter, which is the function of the arc position and current.

\subsection{Assumptions and simplifications for arc model}

To reduce the complexity and computation load of the arc model in an LVSD, the following assumptions and simplifications have been adopted.

- The arc column is considered to be in a state of local thermodynamic equilibrium (LTE).

- The arc initial state is modelled as a hot channel in a small gap between contacts which has a homogeneous temperature distribution ( $1 \mathrm{~mm}$ radius and 10,000 K temperature) (Ma et al., 2009).

- The arc gas motion is regarded as a laminar flow.

- Metal erosion and wall ablation are not taken into account.

- Lorentz force generated by nonlinear ferromagnetic material and current path in a quenching chamber is calculated by FEM prior to the arc simulation using the external magnetic flux density as a input parameter applied to the fluid domain in the arc model.

\subsection{MHD equations in arc column}

The arc column is electrically neutral gas media. It is a mixture of electrons and heavy particles (ions, atoms and molecules) staying in thermal equilibrium at high temperature. If the assumption of LTE holds in the arc column, the arc can be treated as a single fluid and the mass, momentum and energy conservation equations can describe the relation between the velocity, pressure, temperature in the arc column as given below (Karetta and Lindmayer, 1998, Yang et al. 2013),

$$
\begin{gathered}
\frac{\partial \rho}{\partial t}+\nabla \cdot(\rho \vec{V})=0, \\
\frac{\partial\left(\rho v_{i}\right)}{\partial t}+\nabla \cdot\left(\rho v_{i} \vec{V}\right)=-\nabla p+\nabla \cdot\left(\eta \nabla v_{i}\right)+(\vec{J} \times \vec{B})_{i},
\end{gathered}
$$




$$
\frac{\partial(\rho H)}{\partial t}+\nabla \cdot(\rho H \vec{V})=\nabla \cdot\left(\frac{\lambda}{c_{p}} \nabla H\right)+\frac{\partial p}{\partial t}+\sigma E^{2}+S_{r a d}+S_{\eta}
$$

In the equations (6)-(8), $\rho$ is the density, $t$ is the time, $\vec{V}$ is the velocity, $v_{i}$ is the velocity component in $i$ direction, $p$ is the pressure, $\eta$ is the dynamic viscosity, $\vec{J}$ is the current density, $\vec{B}$ is the magnetic flux density, $H$ is the dynamic plasma enthalpy expressed by $h+\vec{V}^{2} / 2, h$ is the static enthalpy determined by $\int c_{p} d T, \lambda$ is the thermal conductivity, $c_{p}$ is the specific heat capacity, $S_{r a d}$ is the radiation energy losses and $S_{\eta}$ is the heat generation due to viscous dissipation. These are standard fluid dynamics equations with an addition of the Lorentz force in momentum equation (7) and ohmic Joule heat plus the radiative losses in the energy equation (8).

The electric field $\vec{E}$, which determines the ohmic heating source in the energy equation, is calculated from Gauss's law, (9) and (10),

$$
\begin{gathered}
\nabla \cdot(\sigma \nabla \Phi)=0, \\
\vec{E}=-\nabla \Phi,
\end{gathered}
$$

where $\sigma$ is the plasma electrical conductivity and $\Phi$ is the electric scalar potential.

The simplified net emission coefficient method is employed in this work to calculate the radiation energy loss $S_{r a d}$, and the net emission coefficients are computed from (11),

$$
\varepsilon=C_{1}\left(\exp \left(C_{2} T\right)-\exp \left(C_{2} T_{r}\right)\right)
$$

where $C_{I}$ and $C_{2}$ are the constant coefficients $300 \mathrm{~W} / \mathrm{m}^{3}$ and $0.0011 \mathrm{~K}^{-1}$ respectively, $T_{r}$ is the ambient temperature and $T$ is the arc temperature (Barbu et al., 2012).

\subsection{Modelling domains and boundary conditions}

Figure 12 shows the domains and boundaries for the FTA's arc model. The fluid component is the internal air volume of the FTA and it modelled by MHD theory with the external magnetic flux density, whereas the metal solid parts are considered as domains including only the electric and heat transfer phenomena. The current density calculated by the load circuit equation (2) is applied to the current-in boundary while there is $0 \mathrm{~V}$ electric potential at the current-out surface. The opening condition is set as atmospheric pressure and temperature (1 atm, $300 \mathrm{~K}$ ). The simulation includes the temperature and pressure dependant properties of air (density, electrical conductivity, thermal conductivity, the specific heat capacity and viscosity) (Murphy, 1995, 2012).
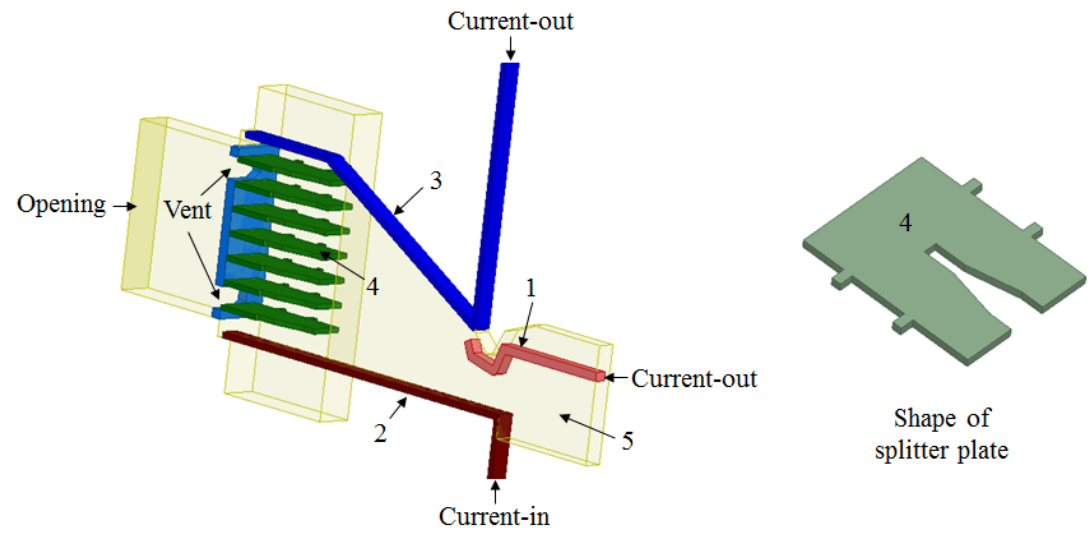

Shape of splitter plate

Figure 12. Domains and boundaries for arc simulation of MCB: (1) moving contact (copper), (2) fixed contact (copper), (3) arc runner (copper), (4) splitter plate (iron), (5) chamber volume (air).

\subsection{Numerical model for arc root}

The arc root is considered as a thin layer between the arc column and the metal surface of the cathode or anode. Before entering the splitter plates, the arc gradually bends and stretches around the plates generating the high voltage necessary to form arc roots on the splitter plates. After arc root formation, the voltage drops in the arc roots on the cathode and anode are relatively high when compared to that in the arc column. This arc root formation determines the exit-voltage and plays an important role in the switching performance of LVSDs. In 
order to consider the arc root formation, further consideration is required as LTE condition does not hold in the arc root region and ordinary MHD theory cannot simulate the arc root phenomena.

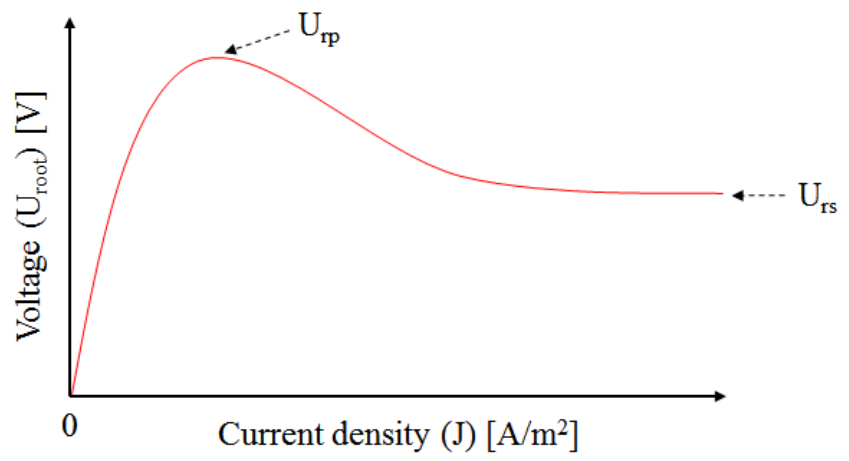

Figure 13. Relationship between potential drop and current density in the arc root.

In order to take into account the arc root formation, the empirical relationship (Lindmayer et al., 2006) between the potential drop and current density is modelled in the simulation tool (see Figure 13). The contact resistance, $C R_{\text {root }}$, considering the effect of the arc root region is adopted on the surface of the splitter plate and it is obtained from (12),

$$
C R_{\text {root }}(J)=\frac{U_{\text {root }}(J)}{J}
$$

where $U_{\text {root }}$ is the potential drop in the anodic and cathodic arc roots. $U_{r s}$ is selected to be $12.25 \mathrm{~V}$ that is a half of both anodic and cathodic drops on the surface of the plate (Nakayama et al., 2003), and the peak voltage $U_{r p}$ across the root is $2 U_{r s}$.

\subsection{Implementation of arc simulation}

The arc modelling process constitutes procedures of arc ignition, MHD computation including the external magnetic flux density, calculation of the external load circuit and contact motion (see Figure 14). After arc ignition, the arc voltage is calculated taking into account the external circuit and contact motion until the first current zero point. This modelling has been implemented in the Ansys CFX commercial software package widely used in previous studies (Lindmayer et al., 2006, Barbu et al., 2012).

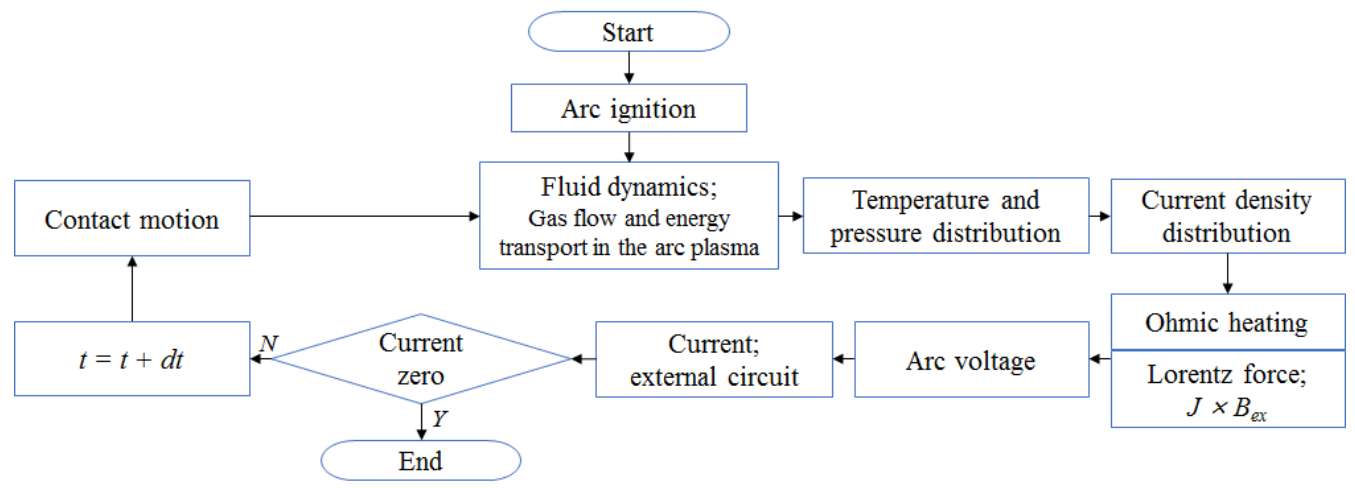

Figure 14. Diagram of the arc modelling process.

\subsection{Validation of arc simulation}

Figure 15 shows the waveform comparisons between experimental and simulated results of the FTA. Figure 16 presents the arc motion data recorded by the arc imaging system and the predicted arc temperature distribution in the quenching chamber, with corresponding voltage and current waveforms in Figure 15. In general, it can be said that the computed waveforms and arc motion have the similar trend with the experimental data. Furthermore, it is worth that this arc model can gives the similar value of the exit-voltage compared to the measured one (simulated value: $252 \mathrm{~V}$, measured value: $280 \mathrm{~V}$ ) 
In Figure 15, the simulated arc voltage is too high at the beginning of the calculation because the thin hot conductive channel is placed between contacts for the ignition modelling but the arc voltage quickly reverses to a low value as the arc develops. As the arc moves towards splitter plates and elongates, the voltage begins to rise. Before $1.5 \mathrm{~ms}$, the rate of change of the simulated current is higher than experimentally observed. This is due to the modelled arc voltage being lower than the experimentally observed value. This is reversed after $1.5 \mathrm{~ms}$ where the modelled arc voltage is greater than experimentally observed leading to a more rapid onset of the current zero point in the simulated case.

In Figure 16, following arc ignition, the arc travels towards the splitter plates. When the arc enters the splitter plates, a high value of arc voltage is generated by the effects of arc root formation. It can be seen that the simulated arc moves faster after $1.65 \mathrm{~ms}$ and reaches the splitter plates more rapidly than experimentally observed. This higher velocity motion leads to a higher arc voltage than measured voltage after $1.5 \mathrm{~ms}$ (see Figure 15). In addition, arc back motion is observed at around $2.25 \mathrm{~ms}$ in both simulated and measured data but its effect in the simulation is reduced when compared with the real arc (the arc moves slightly back and the drop of the arc voltage is small in the simulation compared to the experimental data).

Some discrepancies between the simulation and experiment may be expected from the use of a simplified model of Lorentz force, radiation cooling effect and the absence of metal erosion (which is important when the arc interacts with the plates).

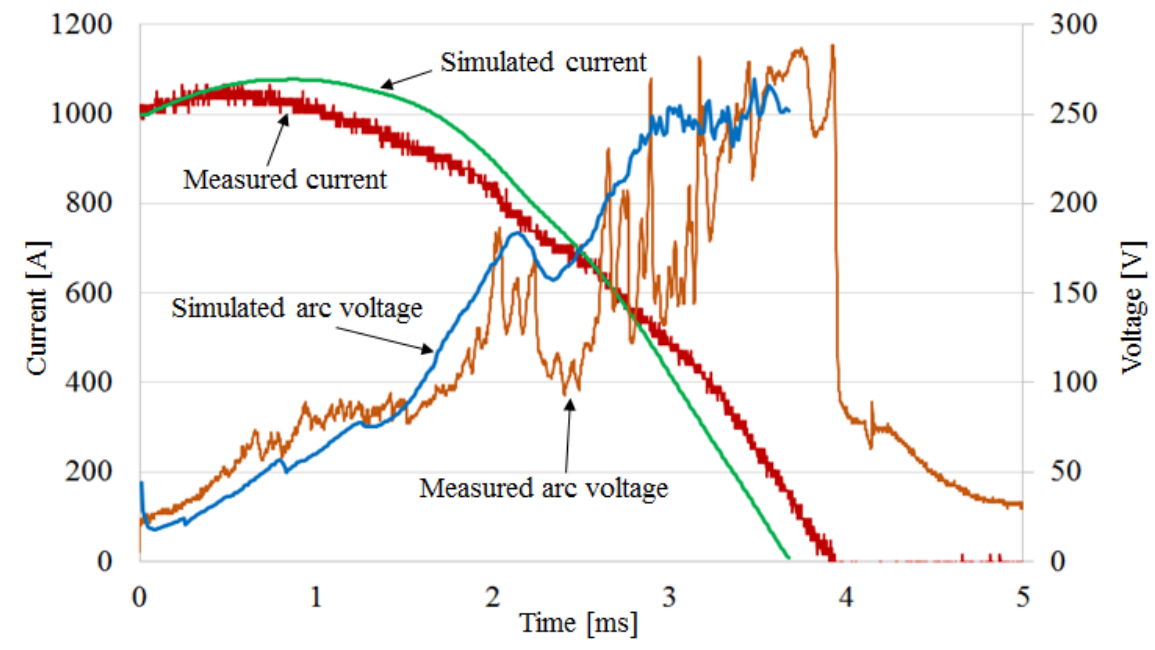

Figure 15. Comparisons between experimental and simulated results of the FTA.
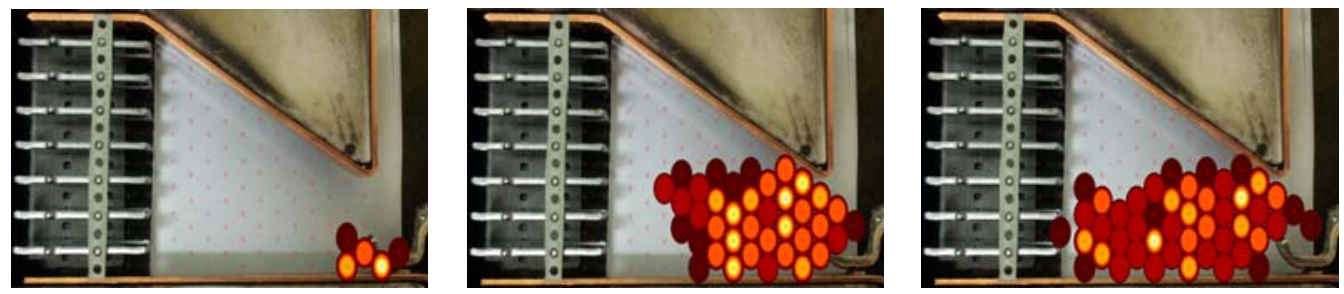

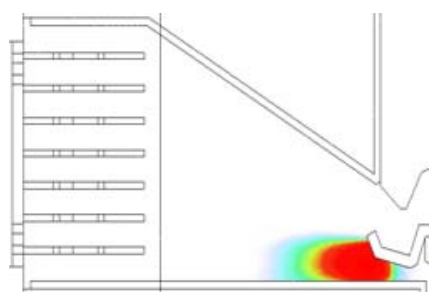

(a) $0.25 \mathrm{~ms}$

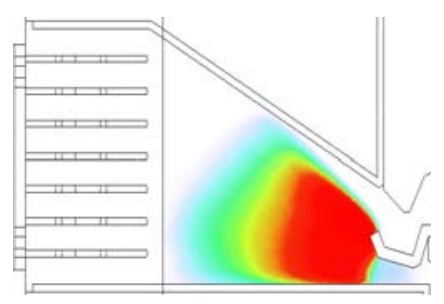

(b) $0.70 \mathrm{~ms}$

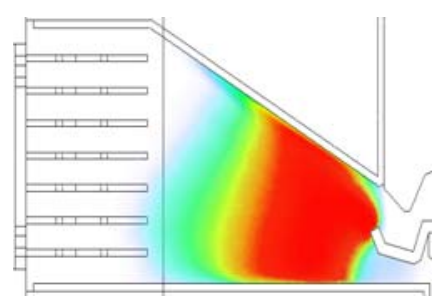

(c) $0.90 \mathrm{~ms}$ 

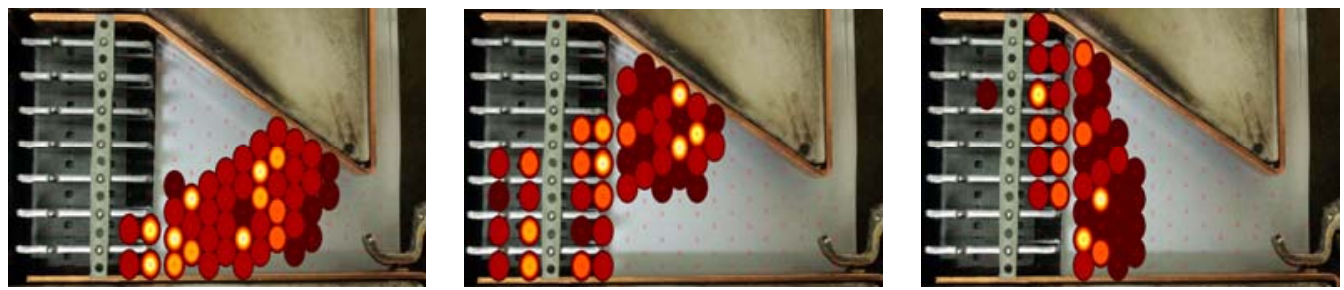

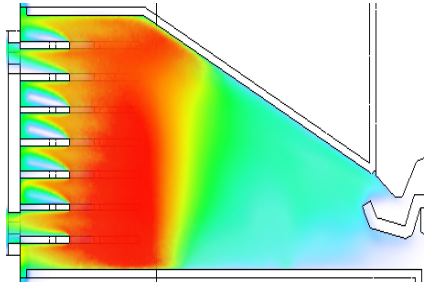

(d) $1.65 \mathrm{~ms}$
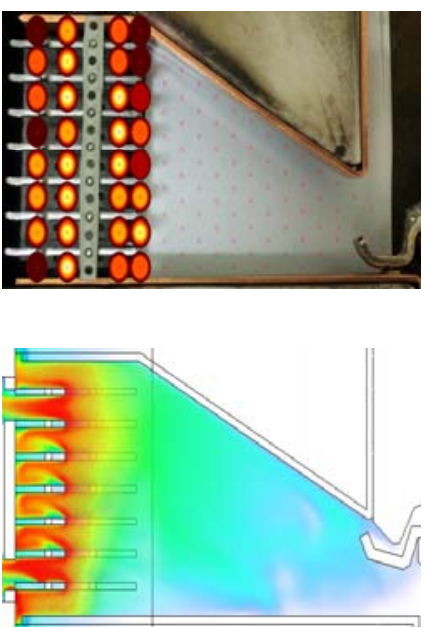

(g) $2.75 \mathrm{~ms}$

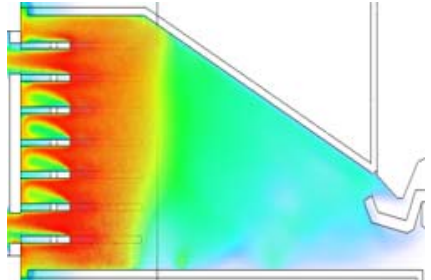

(e) $2.00 \mathrm{~ms}$
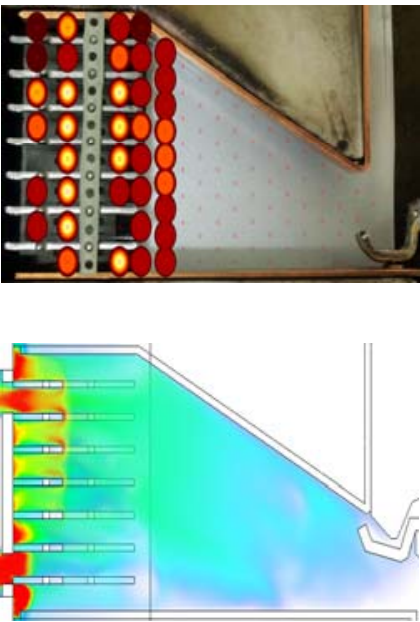

(h) $3.00 \mathrm{~ms}$

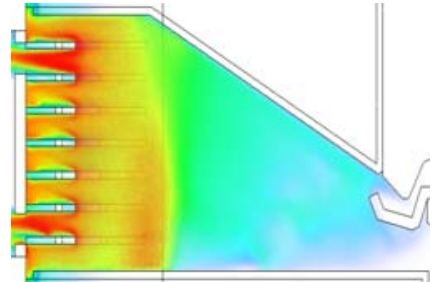

(f) $2.25 \mathrm{~ms}$
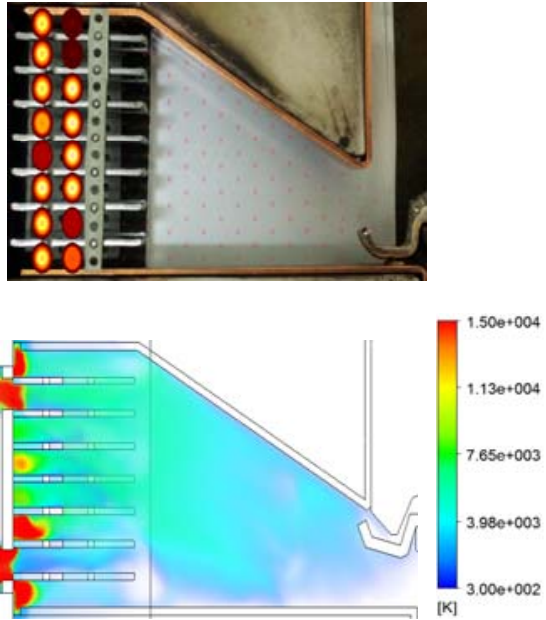

(i) $3.30 \mathrm{~ms}$

Figure 16. Arc images measured by the AIS and simulated temperature distribution (volume rendering in the half model) in the quenching chamber of the FTA.

\section{Conclusions}

The experimental and numerical investigations on the arc behaviour and re-ignition evaluators of LVSDs have been studied in this paper. The following conclusions can be drawn:

- It is found that the ratio of the system voltage to exit-voltage is a reliable evaluator. If the voltage ratio is positive, instantaneous re-ignition never happens. In the case of the negative voltage ratio, if it lies in a range between -1.3 and 0.0 , the re-ignition probability is very low because in such cases the voltage source does not have enough power to support the arc. This voltage ratio can be used for more accurate prediction of re-ignition in the LVSDs without complex calculation of the breakdown voltage.

- The model shows a good agreement with the experimental data. The arc modelling tool can predict the arc voltage and arc current as well as arc motion. Also, the exit voltage is simulated as a similar value with the measured one, which is an important parameter to obtain the evaluator of re-ignition.

- To make better predictions of arc behaviour and evaluation of switching performance, the arc modelling needs to be modified by including the metal erosion effect and improving Lorentz force and radiation cooling calculation.. This advanced fully coupled simulation is a part of ongoing research. 


\section{References}

Balestrero, A., Ghezzi, L., Popov, M. and Sluis, L. (2010), "Current interruption in low-voltage circuit breakers", IEEE Transactions on Power Delivery, Vol. 25, No. 1, pp. 206-211.

Barbu, B., Iturregi, A., Berger, F. and Torres, E. (2012), "Numerical analysis of the electric arc simulation using Ansys CFX", Proceedings of the 26th International Conference on Electrical Contacts, Beijing, China, 2012, pp. 311-316.

Freton, P. and Gonzalez, J. J. (2009), "Overview of current research into low-voltage circuit breakers", The Open Plasma Physics Journal, 2, pp.105-119.

Hauer, W. and Zhou, X. (2014), "Re-ignition and post arc current phenomena in low voltage circuit breaker", Proceedings of the 27th International Conference on Electrical Contacts, Dresden, Germany, 2014, pp. 398-403.

Karetta, F. and Lindmayer, M. (1998), "Simulation of the gasdynamics and electromagnetic processes in low voltage switching arc", IEEE Transactions on Components, Packaging, and Manufacturing Technology, Vol. 21, No. 1, pp. 96-103.

Lindmayer, M., Marzahn, E., Mutzke, A., Rüther, T. and Springstubbe, M. (2006), “The process of arc splitting between metal plates in low voltage arc chutes", IEEE Transactions on Components and Packaging Technologies, Vol. 29, No. C, pp. 310-317.

Ma, Q., Rong, M., Murphy, A. B., Wu, Y. and Xu, T. (2009), "Simulation study of the influence of wall ablation on arc behaviour in a low-voltage circuit breaker", IEEE Transactions on Plasma Science, Vol. 37, No. 1, pp. 261-269.

McBride, J. W., Pechrach, K. and Weaver, P. M. (2002), "Arc motion and gas flow in current limiting circuit breakers operating with a low contact switching velocity", IEEE Transactions on Components and Packaging Technologies, Vol. 25, No. 3, pp. 427-433.

Mcbride, J. W., D. Shin, and T. Bull (2016), "A study of the motion of high current arcs in splitter plates using an arc imaging system", Proceedings of the 28th International Conference on Electrical Contacts, Edinburgh, GB, 2016, pp. 175-180.

Murphy, A. B. (1995), "Transport coefficients of air, argon-air, nitrogen-air and oxygen-air plasmas", Plasma Chemistry and Plasma Processing, Vol. 15, pp. 279-307.

Murphy, A. B. (2012), “Transport coefficients of plasmas in mixtures of nitrogen and hydrogen", Chemical Physics, Vol. 398, pp. 64-72.

Nakayama, K., Yokomizu, Y., Matsumura, T., Kanamori, E. and Kuwamura, K. (2003), "Mechanism of voltage rise of high-current arc at atmospheric pressure due to deion plates", Electrical Engineering in Japan, Vol. 145, No. 3, pp. 17-24.

Rong, M., Ma, Q., Wu, Y., Xu, T. and Murphy, A. B. (2009), “The influence of electrode erosion on the air arc in a low-voltage circuit breaker", Journal of Applied Physics, 106, 023308.

Shin, D., Golosnoy, I. O. and McBride, J. W. (2015), "Numerical analysis of switching performance evaluators in low-voltage switching devices", in International Symposium on Electromagnetic Fields in Mechatronics, Electrical and Electronic Engineering, Valencia, Spain, 2015.

Shin, D., Golosnoy, I. O. and Mcbride, J. W. (2016), "Arc modelling for switching performance evaluation in low-voltage switching devices", Proceedings of the 28th International Conference on Electrical Contacts, Edinburgh, GB, 2016, pp. 41-46. Slade, P. G. (2014), Electrical Contacts Principles and Applications, CRC Press, New York.

Slepian, J. (1928), "Extinction of an A-C. arc", Transactions of the American Institute of Electrical Engineers, Vol. 47, No. 4, pp. $1398-1407$.

Yang, F., Wu, Y., Rong, M., Sun, H., Murphy, A. B., Ren, Z. and Niu, C. (2013), "Low-voltage circuit breaker arcs - simulation and measurements", Journal of Physics D: Applied Physics, Vol. 46, No. 27, 273001. 